\title{
How Students Create Verbal Descriptions of Physical Parts
}

\section{Dr. Steven C. Zemke, Gonzaga University}

Steven Zemke, Ph.D., has been involved in engineering design and teamwork for over 35 years as a professional engineer, university professor, and researcher. He is a Professor of Mechanical Engineering at Gonzaga University in Spokane, Wash., and teaches engineering design and teamwork courses. His two research foci are how students learn design and teamwork and how understanding of learning can be used to create more effective pedagogy. Prior to teaching, Dr. Zemke was a professional product designer for over 20 years with an emphasis on mechanical packaging of microwave circuitry.

\section{Dr. Diane L. Zemke}

Diane Zemke is an independent researcher and consultant. She holds a Ph.D. in leadership studies from Gonzaga University. Her research interests include teamwork, small group dynamics, dissent, organizational change, and reflective practice. Dr. Zemke has published in the International Journal of Engineering Education, the Journal of Religious Leadership, and various ASEE conference proceedings. She is the author of "Being Smart about Congregational Change." 


\section{How Students Create Verbal Descriptions of Physical Parts}

Clear and precise communication is a fundamental skill that professional engineers need. They employ it while doing engineering, such as interacting on design teams, and while communicating engineered results, such as in technical reports. Furthermore, communication comes in many forms, such as written reports, verbal interactions, documented calculations, and engineering drawings. Consequently, "an ability to communicate effectively," is a required student outcome for accreditation in ABET criterion 3g. ${ }^{1}$

This current study examines the verbal aspects of communicating design information. Whereas textbooks in design extensively describe graphical communication, they are fairly silent on how to verbally describe parts. For example, Technical Drawing by Giesecke et al. thoroughly describes graphical communication of parts, but does not mention verbal descriptions. ${ }^{2}$ The Mechanical Design Process by Ullman briefly mentions that parts can be described semantically and that teams must communicate to collaborate, but does not elaborate on semantic representations. ${ }^{3}$ Verbal descriptions of parts seem to lie outside the typical curricular materials for engineering.

In a previous study we identified that students had difficulty communicating design ideas with their peers on design teams. ${ }^{4}$ This difficulty occurred during sketching and verbal descriptions of parts and assemblies. Students' difficulties with verbal communication directly interfered with their ability to work productively together.

In this study we examine the verbal aspect of students communicating about parts and assemblies. The students were asked to describe parts and assemblies of common hardware store items that were physically in their hands. We assumed that describing a physical part in hand is fundamental to being able to describe any part, whether it is in a drawing, a CAD system, or simply being conceived of.

The intent of this study was to learn how students create descriptions of physical parts and assemblies. Hence the driving question in this study was:

What characterizes students' descriptions of parts and assemblies that they are physically examining?

This qualitative multiple case study was conducted in an engineering school where seven students were video-recorded while describing three separate hardware store items. The video data was analyzed using standard content analysis methods and rich descriptions were written of each student's approach to the task. A further rich description was written describing the common trends in descriptions across multiple students. The analysis showed that students use a variety of approaches to describe the parts, each approach has strengths and weaknesses, and that every student found the task difficult. 


\section{Background}

Being able to communicate verbally about one's design is key to being able to design, particularly if designing occurs within a group. ${ }^{5}$ When that ability to verbally communicate is poor, the ability to design is negatively impacted.

In our prior study we examined students' learning journals, where they reflected on their experiences in teamwork and design. ${ }^{4}$ That study indicated that poor verbal communication formed a significant hindrance, both in design and teamwork. This inability to communicate was widespread. For example, one student noted:

Trying to explain a part, or having a part explained to you, solely in words is almost impossible.

Another student noted difficulty as well:

Routinely one of us will come up with an idea, and then try to explain it and the team will imagine something almost completely different. ${ }^{4}$

These students were fluent in a common language (in this case, English) as well as sharing common coursework that should have helped them to converse about their designs. Yet they obviously struggled to verbally communicate regarding their designs. There appear to be several bases for these difficulties.

Bucciarelli argued that different design disciplines manifest different "object languages."6 These object languages are based in discipline-specific conceptual worlds. Thus, different engineering disciplines have different object languages, which can make interdisciplinary work challenging. These object languages consist of discipline-specific terms and the understanding of these terms and discipline-specific norms, such as the meaning of "quality." They will include relevant formulas, time scales, and metaphors. In short, communicating about one's design effectively means that one must be adept with the relevant object language.

The difficulty with an object language is that it masquerades as one's own native language. For example, since one is using the object language to create descriptions in English, one is tempted to think that everyone is understanding the description because it occurs in English. One does not realize that the description is actually occurring in the object language. ${ }^{6}$ Students or novices who may not be adept at the object language fail to understand what is being described. Similarly, students, lacking skill in the object language, will struggle to create descriptions that make sense to others within that object language.

Wiegers et al explored the idea of object language from a different perspective, studying how people verbalized shapes. ${ }^{7}$ This ability to verbalize shapes and their orientations is key to describing mechanical parts and assemblies. Wiegers et al noted that the description of shapes is replete with errors. These errors arise because the speakers' image of the shape is unclear or they use the wrong word or grammar. Speakers can be inconsistent or are working from a different frame of reference. What the speaker is seeing or visualizing is not accurately conveyed to the listener. $^{7}$ 
Thus, describing shapes alone is a source of difficulty. However, describing mechanical parts and assemblies consists of more than describing shapes. One must also describe orientations, measurements, and sometimes, functions, for a complete and useful portrayal of the part or assembly.

Bucciarelli noted that the object language is "learned on the job as well as in disciplinary coursework within schools of engineering." It appears he would argue that students will acquire the object language even if it is not explicitly taught. Like Bucciarelli, Wiegers et al noted that differences in education affect the way that people describe shapes. ${ }^{7}$ Yet engineering education often does not focus on explicitly teaching the relevant object language to students. Instead, it is hoped students will acquire what is needed along the way. This hope is not always borne out. Students and instructors vary in their ability to convey and learn tacit or implicit skills. ${ }^{8}$ Furthermore, even with explicit instruction, people can fail to learn because of prior misconceptions. ${ }^{9}$

Schön delineated between the skills of novices and those of professional practitioners. ${ }^{10}$ Experts had rich "repertoires" of terms, understandings and metaphors. In short, they would be able to communicate well within their object world. This repertoire is built up over time, through instruction and, more importantly, varied professional experience. In contrast, students' or novices' repertoires are relatively empty. They lack the terms and conceptual understanding that experts have. They have not had enough time, nor the varied professional experience, to develop the repertoires they need. It is these repertoires that undergird the ability to describe parts and assemblies and enable the object language to function.

However, repertoires are not filled only by classroom instruction. Instruction and testing over technical terms and formulas fills one part of the repertoire; it does not complete it. Our previous research revealed that students stock their repertoires in a number of ways, one of which was hands-on learning. ${ }^{4}$ For example, in that study, a student noted:

In this case we were given a list of materials we could use for our project. The problem is feeling a list doesn't really help me. I need to feel and see the materials in order to fully understand their capabilities as well as their limitations. ${ }^{4}$

Stocking a sufficient repertoire is based on all the students' experiences in the domain, which includes coursework, past experience, extracurricular design experience, and so forth.

Thus, effective verbal communication is challenging on several fronts. Students have not acquired the necessary object language, they, like many, struggle to describe shapes, and they lack sufficient repertoire.

\section{Study context and methods}

This study was conducted in a private undergraduate university within its Engineering School under the approval of the Institutional Review Board. The seven students who volunteered to participate, four males and three females, were Mechanical Engineering juniors within a required design course. 
Each student was asked to describe three common items from a hardware store: The first was a low-flow garden sprinkler assembly, the second was a steel electrical junction box, and the third a pair of slip joint pliers. These items are shown in Figures 1-3. Two copies of each item were presented to the students, one fully assembled and one disassembled. The disassembled item allowed the student to examine and describe features that would otherwise be obscured by assembly. The student was then prompted describe the item as they would to another engineer. If the student had questions about the task, either at the beginning or during it, the researcher would answer the questions concerning the task.

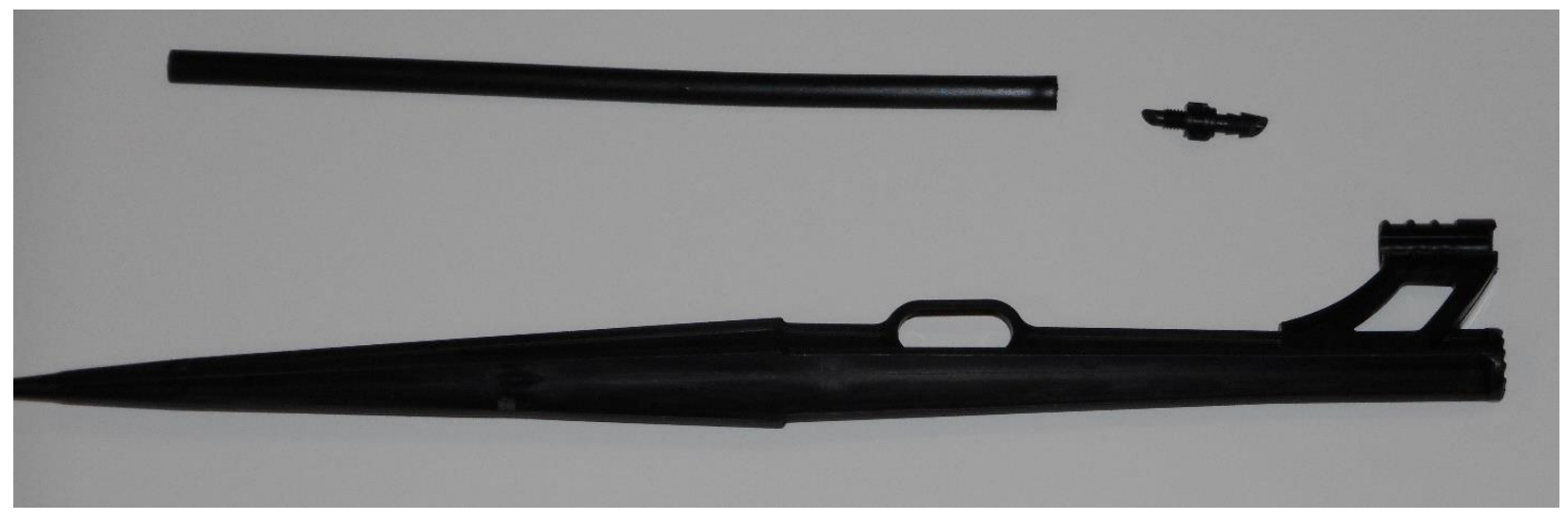

Figure 1: Garden sprinkler parts, unassembled.

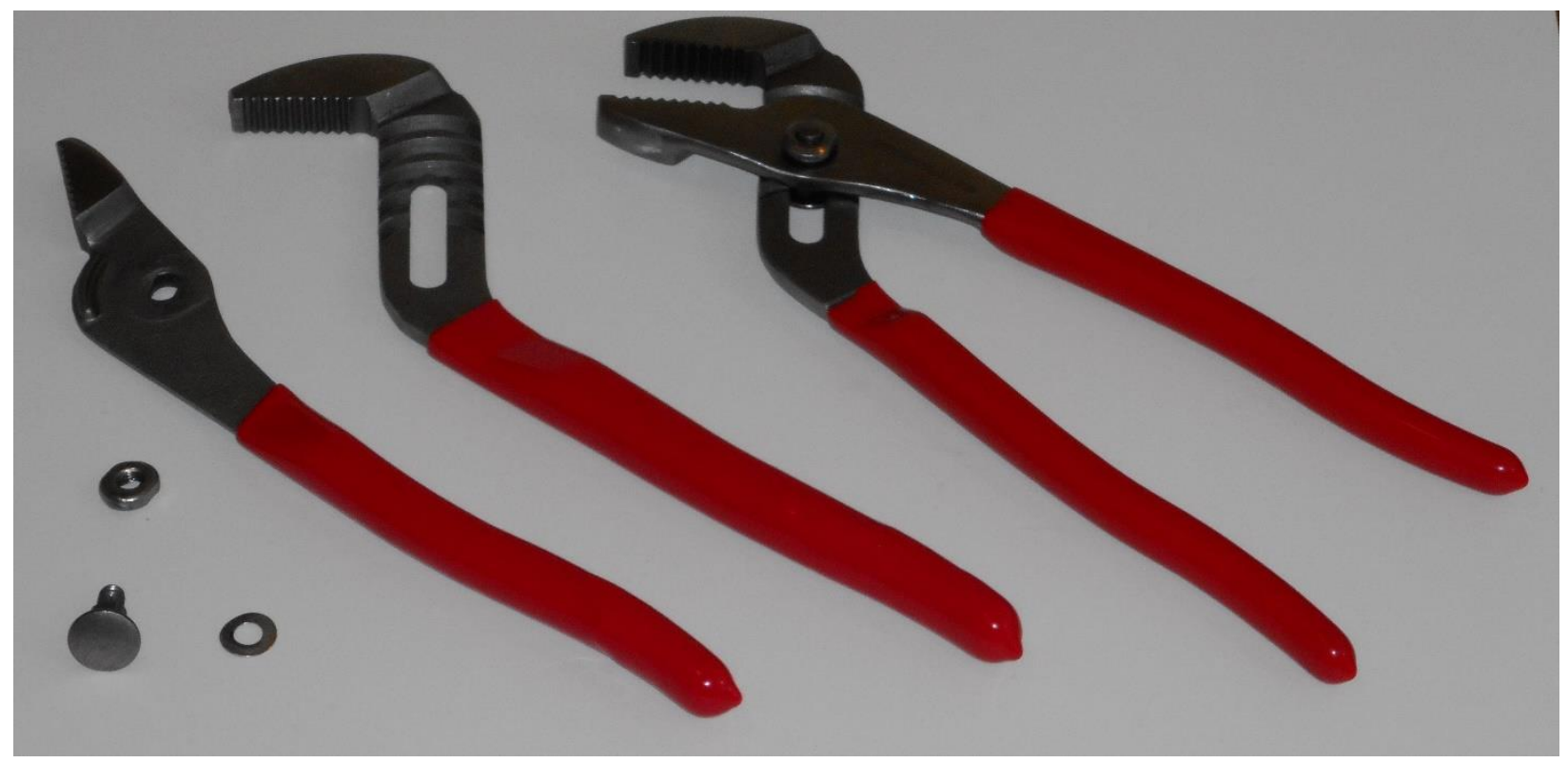

Figure 2: Slip joint pliers, assembled and unassembled. 


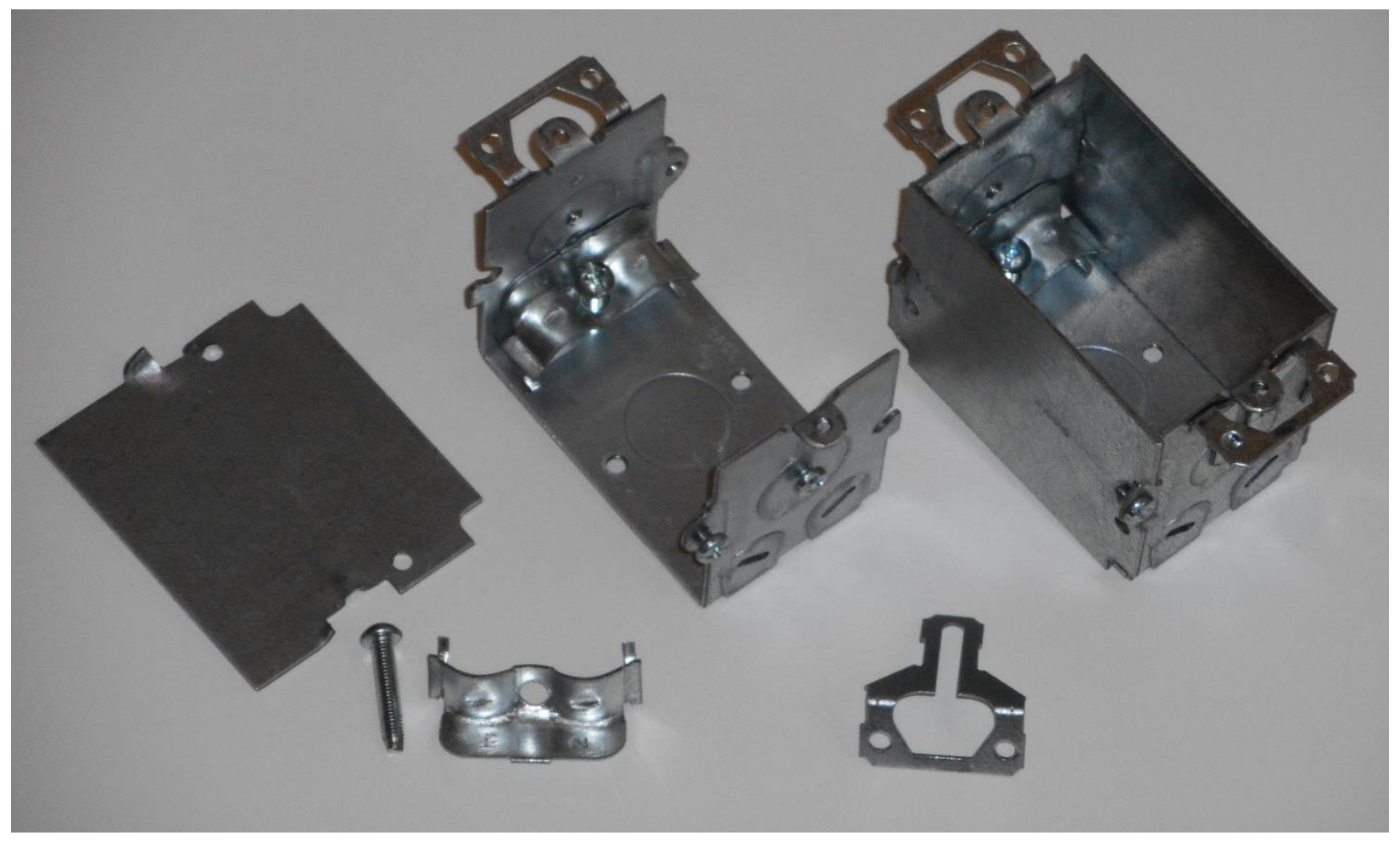

Figure 3: Steel electrical box, assembled and unassembled.

Each student was video-recorded with only the researchers present. When the recording began, each student was presented with the first item. The recording was ended when the student verbally concluded his or her description. The student was given a second item to describe, and then a third. All students were given the items in the same order. Each student described all three items, resulting in 21 separate video-recordings. The video-recordings form the data in this study.

We used qualitative methods for this study. Since this study was exploratory, a descriptive approach seemed most appropriate. ${ }^{11}$ Since we were studying students' verbal communication, being able to create rich descriptions was important. ${ }^{12}$ Qualitative research employs a wide variety of methods. We selected a multiple case study approach. Case studies examine bounded, integrated systems and multiple case studies often have small sample sizes. ${ }^{13,14,15}$ Each student constituted a case.

However, a case study method does not specify how the data is to be coded. Since the data consisted of videos (and subsequent transcriptions) we used content analysis to code for manifest (or obvious) themes. ${ }^{10}$ Content analysis is used to examine the content and meaning in texts. The term text is used broadly and refers to documents or communication "produced by someone to have meanings for someone else." 16 Transcriptions of the video recordings are the text being analyzed in this study. Each transcription was identified by assigning pseudonyms to the students.

When all recordings were completed, they were transcribed word for word. Unusual or extended gestures or silences were annotated. These transcriptions were then coded using inductive 
content analysis methods. ${ }^{17,18}$ These methods identify themes within the data and subsequently code the data into these themes. A rich description was then written to characterize each participant. During the writing of rich descriptions, the videos were reviewed in tandem with the transcript to insure fidelity to the coding themes. The following themes were identified.

- Geometric Description: The student described the part or feature in terms of geometric shapes and/or specific measures of size.

- Technical Vocabulary: The student used a technical term to identify a part or feature in a single statement.

- Functional Description: The student described the function of the part or feature (how it worked) instead of describing the part (what it was).

- Modeling Description: The students described the part in terms of how to 3D model the part or how to physical make the part.

- Analogical Description: The students described the part as analogous in shape to an unrelated physical item.

- Demonstrate Difficulty: The student expressed or otherwise demonstrated that describing the part was difficult.

\section{Results}

Based on the transcripts and on observing the video-recordings, we have created rich descriptions of each participant's strategies. Initially, we thought we would be able to create a rough sketch of each assembly based on each student's descriptions. This strategy proved impossible for all participants and for all assemblies.

\section{Adam}

Geometric terms provided the primary framework for Adam's descriptions. In each case he relied on geometric terms and at times they seemed to echo terms learned in CAD modeling. For example, during the sprinkler description, he stated "it's a cross that meets a circle but without the perimeter, just the cross." With the electrical box he began with "it's like a cube with one end open."

Although he did describe each component, he often missed key features of individual components. And this problem was not related to the complexity of the individual part. Adam also relied on perceived functions to leverage descriptions. When the function was clear, the description was clearer. Descriptions wavered when the function was unclear.

Additionally, Adam relied on some analogies to describe parts. "And at one end it comes up to a ... so much like a . . back end of a plane. Just a wing. . . " Or, "grooves cut into it to fit it into place and kind of like a puzzle manner."

Adam did use the appropriate technical terms occasionally, such as flange, flat head screw, groove, nut, and interlocking. He also occasionally used precise measurements such as $90^{\circ}$ or one inch. However, overall, he did not use many measurements, technical terms, or specific terms describing orientations. 
Adam openly stated his inability to describe parts in each case. He struggled with geometric terms. "It's a circle with, ah, the shape escapes me but, ah, elliptical is the closest one I can think of." He struggled with technical terms: "I think it's a rivet if I remember right, but I don't know." And, he struggled with missing information: "I can't think of the name of it." He also noted his difficulty in describing parts. "There are a lot of intricacies to this part" when referring to the electrical box. He stated "It's hard to describe" a portion of the sprinkler assembly.

\section{Brad}

Overall, Brad seemed less assured in his descriptions than Adam. His manner was slower and more tentative, with silences or short aborted attempts: "The next part . . . is a . . looks like we'll just start with the handle. There's a handle on it" (referring to the slip joint pliers). Brad also verbalized his inability to describe parts and assemblies: "That's about all I would describe because I don't know how to describe the other parts."

Brad organized each of his descriptions by starting with the complete assembly and stating its purpose: sprinkler, electrical box, or slip joint pliers. Analogies were Brad's strongest tool. One arm of the slip joint pliers was a "hockey stick" and the other had a tip like a "bird beak." The sprinkler assembly had a "gun shape." The wire clamp in the electrical box looked like a "sine function."

His other tool was substituting perceived functions for descriptions. When describing a flange on the electrical box, he noted, "One of the last pieces looks like the last part put into the actual beam in the wall." He did not describe the flange itself. Similarly, "Another feature it looks like a ... is fastened with a screw and it can slide up and down on the screw." Again, the function substituted for the description.

Brad was not thorough in his descriptions. With the slip joint pliers he only described the two arms, completely neglecting the bolt, nut, and washer. Parts of the electrical box were also ignored.

Brad described materials and processes with the electrical box, such as "a two-fold sheet metal part." He used geometric terms such as perpendicular and pyramid, as well as technical terms such as flange, screw, and male/female ends. However, he used few measurements.

\section{Carol}

Carol verbalized her difficulty in describing parts throughout. She missed terms: "The bolt doesn't have an ... shoot ... word ... ha ha. Yah, there's no slot. . .." (after one of the researchers supplied the term.) Regarding the sprinkler, she ended her description saying "I don't really know what to say." She concluded her electrical box description that same way.

Understanding function was very important to Carol. With the slip joint pliers she spent time using the assembly and understanding how it worked. Similarly, with the sprinkler assembly, she repeatedly snapped the parts together. Carol used this understanding of function to leverage her descriptions. When she didn't understand a function, she directly asked: "Sorry, what did this one do again?" Or, "I guess I'm still confused by what this does." Even when refocused by the researchers to describe the parts, she returned to understanding the function and using the 
components. She noted her difficulty in describing without understanding the function: "This part ... I forget what this one does, but it's got threading . ..." She finished describing this part (a coupler for tubing) by saying "It looks like it's meant to fasten rather than perform any functions."

Carol was thorough, describing each component in each case, but the level of description varied. She was very specific about a screw in the electrical box: "A screw, simple, inch long, it's got an incline, it's got threading on it, it's got a Philips head." However, another component was described as a "weird smaller piece with two little off shoots that are bent . . . A lot of it's rectangular." Or, she noted the box had "various holes for screws and wires" without providing more information.

Carol did use some analogy but it was less pronounced. She also used a compare and contrast strategy with the arms of the slip joint pliers, noting how the arms were similar and different rather than directly describing each arm independently.

Carol used measurements, such as one inch, half a centimeter, and $90^{\circ}$, as well as geometric terms such as semicircular and rectangular. She also used technical terms such as face, threading, slot, incline, bolt, and collar.

Dan

Dan was very thorough in his descriptions. He began each description by stating the number of components involved. He then moved through the components in an orderly fashion, completing one before moving to the next.

Unlike other students, Dan did not use analogies to describe parts. Nor did he leverage function. He did occasionally mention the function of an individual component, but that function was not related to the description. Nor did he rely on CAD modeling terminology. Instead, Dan's descriptions were replete with measurements, geometric terms, and technical terms. His descriptions were relentlessly detailed. Initially, we thought we would be able to sketch parts based on Dan's descriptions. However, the descriptions were so focused on individual attributes of parts without noting relative position to other attributes that sketching proved impossible.

Like other students, Dan verbalized how challenging this task was. There were some heavy sighs during descriptions. He stated the slip joint pliers were "very hard to describe" at the outset. With the sprinkler, he sighed and asked, "How would I describe that," but then proceeded to give a thorough description.

\section{Elaine}

Elaine spent time studying each assembly and its parts before beginning. In each description she was careful to state the name of the assembly (e.g. electrical box) and the number of components.

However, Elaine struggled with providing even minimal descriptions and her ability did not improve as time went on. In each case the researchers verbally prompted her to describe the 
parts, but she continued to discuss function and how to construct the assemblies. She appeared to be incapable of providing even simple descriptions.

Elaine used very few technical terms, measurements, or geometric terms. She used no analogies. She did use one term from CAD modeling, which was "extrude" when referring to a raised ridge on one of the arms of the slip joint pliers. Otherwise, she was completely focused on the function of individual parts and how they went together.

Although Elaine exhibited the poorest ability to describe parts, she never noted that the task was challenging.

Fay

On her way to begin the task Fay noted that she was very poor at descriptions. However, during the task she did not state that the task was difficult nor demonstrate it nonverbally with sighing. Fay's descriptions were rapid and assured, though not complete enough from which to sketch the parts.

In each case Fay spent time building the assembly from the various components. As she built the assembly she described each component. While Fay did describe each component in each case, the descriptions often used indefinite terms or were superficial. For example, the slip joint pliers were described as having "red rubber handles and they're silver" without describing the shape of the arms. Or, referring to the stake component in the sprinkler: "But then at the center it's bigger and then it becomes smaller toward the end of it."

CAD modeling terminology entered some of Fay's descriptions. The most common word was "extrude" to refer to any feature coming out of the part. The slot on the sprinkler stake was "extruded." The ridge on one arm of the slip joint pliers was "extruded" as well.

Fay occasionally used some analogies. The slip joint pliers "open and close like scissors." Part of the sprinkler stake resembled "an airplane wing. Or like the tail of an airplane." However, analogies were not frequent.

Nor did she use function as a way to leverage or substitute for descriptions. When she mentioned function, it was directly related to constructing the assembly itself.

Fay did use technical terms occasionally such as "key cutout, square cutouts, and died end." However, she struggled with accuracy. Screws and bolts were referred to as "nails." Fay also used some geometric terms such as "cylinder" and measurements such as "one inch" or "one centimeter." Overall, though, she did not rely on measurements.

\section{George}

George spent a lot of time studying parts and assemblies. With the electrical box, he spent 40 seconds looking at the part and whispering "um, describe the part." During this time he attempted to assemble part of the box. With the sprinkler assembly, he ended by studying the stake for 24 seconds before saying "I think that's about it." Of all the students he spent the most time silently or nearly silently studying each part. 
Although he didn't express difficulty, he did exhibit that the task was difficult. With the wire clamp in the electrical box he stated "It's a very complicated awkward part. It looks like it has two shallow [leaving his thought incomplete and not returning to it]. One, one of the sides is flat ...." He also used restatements: "It looks like there is a slot . . it looks like there is a slot in these pieces ...."

He did use some analogies, but analogy was not a common tool. He leveraged function in two ways. The first, and far more common, was to explain how the component functioned in the assembly. "The stopper controls how far the hose goes on." Occasionally, he used function to supplement the description: "[It] has grips on it that are used to grip whatever the wrench is holding." However, he did not always get the function right. He consistently referred to the coupler in the sprinkler as a "nozzle."

George's descriptions were thorough and replete with measurements, geometric shapes, and technical terms. He was the only student to describe the hose in the sprinkler assembly with measurements and features. However, his technical terms were not always correct.

\section{Summary of data}

The participants used many of the same verbal description methods, however each differed in how much he or she relied on any specific method. Table 1 summarizes each participant's reliance on each method.

\begin{tabular}{|l|c|c|c|c|c|c|}
\hline & $\begin{array}{c}\text { Demonstrate } \\
\text { Difficulty }\end{array}$ & $\begin{array}{c}\text { Geometric } \\
\text { Description }\end{array}$ & $\begin{array}{c}\text { Technical } \\
\text { Vocabulary }\end{array}$ & $\begin{array}{c}\text { Functional } \\
\text { Description }\end{array}$ & $\begin{array}{c}\text { Modeling } \\
\text { Description }\end{array}$ & $\begin{array}{c}\text { Analogical } \\
\text { Description }\end{array}$ \\
\hline Adam & High & Medium & Medium & High & Medium & Medium \\
\hline Brad & Medium & ----- & Medium & High & ----- & High \\
\hline Carol & High & Medium & Medium & High & ----- & Medium \\
\hline Dan & Medium & High & High & ----- & ----- & ----- \\
\hline Elaine & ----- & ----- & ----- & Medium & Medium & ----- \\
\hline Fay & Medium & Medium & Medium & ----- & Medium & Medium \\
\hline George & Medium & High & High & Medium & ----- & Medium \\
\hline
\end{tabular}

Table 1: Relative reliance on description methods by participants.

The data can also be summarized in aggregate terms of how often each of the description methods were used. During coding, each phrase was categorized into the description method category and tallied. The students were on task throughout their descriptions and hence very few phrases did not fit the categories. Table 2 provides these tallies. 


\begin{tabular}{|l|c|c|}
\hline Category & Tally of Occur. & \% of Total \\
\hline Demonstrate Difficulty & 14 & 2.2 \\
\hline Geometric Description & 247 & 39.5 \\
\hline Technical Vocabulary & 183 & 29.3 \\
\hline Functional Description & 124 & 19.8 \\
\hline Modeling Description & 37 & 5.9 \\
\hline Analogical Description & 20 & 3.2 \\
\hline Totals & 625 & 100 \\
\hline
\end{tabular}

Table2: Frequency counts of the description methods in the aggregate student descriptions.

\section{Discussion}

The results section provided a rich description of how each participant constructed descriptions. The following discussion characterizes each description method across all participants. Tallies of how often each method was used are shown in Table 2 as an overview. However, these tallies provide only a surface understanding of what was happening as the students described parts. The following subsections richly describe the characteristics that emerged in the descriptions.

\section{Student difficulties while describing parts}

Direct statements of having difficulty tallied as only $2 \%$ of the utterances, however the observed difficulties ran throughout the descriptions. Every student showed difficulty in describing some parts and some students showed difficulty in describing every part. Long silences, shorter pauses, puzzled stares, and sighs punctuated the descriptions when difficulty was encountered. Further, at times the verbal descriptions became nearly uninterpretable, also indicating difficulty.

The uttered statements of difficulty identified a few different areas of difficulty. Some shapes seemed to defy description. For example, when describing the semicircular ridge with a trapezoidal cross section on the pliers one student said, "This is very hard to describe, ok!" Students expressed difficulty in recalling a specific word or term, "shoot. . . word. . . ha ha!," and difficulty understanding a function, "I'm not sure what it's used for," as well as broad expressions of difficulty, "I'm sorry." Occasionally, a student would pick up a part, examine it, and put it down with little or no verbal description. The primary thing to note is that verbal descriptions were hard for the students. 


\section{The role of understanding of function in describing parts}

Students described the parts in functional terms in about $20 \%$ of the utterances. The commonality in the functional descriptions is that the descriptions do not actually describe the parts or features, but rather how the part or features work. For example, students would describe how the device worked such as, "it locks when you open it all the way." Students would also describe the purpose of parts, such as "connective pieces." Students would describe how the feature on the parts worked together or assembled, such as "slide up and down on the screw."

Two surprising characteristics appeared with the functional descriptions. First, the students had difficulty describing the parts when they could not assemble them. They would first spend significant time attempting an assembly before describing the parts. On some occasions, the students would stop describing a part mid-stream to determine an assembly step before proceeding. On a few occasions the students would substitute a step-by-step assembly sequence rather than describing the parts themselves. In one instance, the student simply could only give an assembly sequence but not a part description, even when prompted. In all these instances it appeared that the students needed to understand how the parts assembled to recognize the shapes on the parts themselves.

Second, the students had difficulty describing the parts if they did not know how they worked or were used. They asked the researcher to explain the part's use or purpose. This behavior was especially true for the sprinkler parts. Frequently, the students would then incorporate the part's use into the description in a describe-shape-then-function formula. This formula was also evident when the students knew the part's function without asking.

In both types of cases where function was leveraged into the description, it appeared to be fundamental to thinking about the parts. It seemed that to describe a part, one had to link the features of the part to an underlying rationale for their existence.

\section{Lack of technical vocabulary and the use of modeling terms}

Technical vocabulary accounted for about $30 \%$ of all coded utterances. Technical vocabulary, similar to geometric terms, conveys information quickly except that it conveys much more complex geometry. For example, the shape of a thread cannot be described easily with geometric terms. Technical terms for both parts, such as bolt, and features, such as thread were used.

The students used incorrect technical terms at times, such as identifying a screw as a nail. On some occasions the students did not know a specific technical term, such as a pocket or a shoulder when it would have added clarity to their descriptions. The students also did not use common drafting technical terms. For example, no students used the term cross-section when describing the shape in the middle of a part. Presumably, the students did not know the terms and hence didn't use them. It seems that adding a brief list of technical terms into a curriculum and then using them may remedy this shortcoming.

Another technical way to describe parts is leveraging how the part is manufactured or modeled. The students used the occasional modeling or machining term; however, they frequently and effectively used the manufacturing term bend while describing the electrical boxes. This particular term allowed them to describe the flat surfaces as they progressed from one point to 
another. It should be noted that all participants were currently enrolled in a design class where every prototype included some sheet metal parts which they had formed.

\section{Misuse of analogy}

Occasionally, students would use an analogy to describe a shape. Some students used this method more than others. For example, "looks like a hockey stick," was used to describe part of the pliers and "the part as a whole is gun shaped," was used to describe the sprinkler support stake. Most analogical descriptions were not clear because the associated parts of the analogy were not specified. In the previous example the analogous features between the pliers and the hockey stick were not made clear.

\section{The overall structure of the student descriptions}

The overall verbal structure of the descriptions was idiosyncratic. However, two thirds of the descriptions began with an introduction. In shorter introductions the students simply listed the number of parts. Some of the introductions were somewhat more complete where the students named either the assembly or the piece parts. The three longest introductions provided an overview of the complete assembly. The students gave an overall size and shape to the assembly, identified parts, and in one introduction the student described how the parts fit together.

The introductions depended on what was being described. All descriptions of the slip-joint pliers began with an introduction. In contrast, only one of the sprinkler descriptions began with an introduction.

\section{Lucidity and an Emerging Taxonomy of Descriptions}

A formal structure for clear verbal descriptions can be constructed from the characteristics of the best descriptions that were observed. First, the best descriptions began with an introduction which described both the assembly and how the parts related to the assembly. An intentional introduction can orient the listener relative to the shape of the entire assembly, its parts, and its function.

Technical vocabulary should take a lead role in descriptions. These terms fulfill a similar role as geometric terms except that more features can be described more precisely with a single term. Notably, the student descriptions were much clearer when they correctly used technical terms. Further, technical terms such as cross-section, which describe orientation, position, or internal shape, add a much needed tool.

Manufacturing processes play a role similar to technical vocabulary. Each manufacturing process constrains parts, such as uniform part thickness and bends in sheet metal, which augments other descriptions elements. The students effectively used the process they knew to clarify their descriptions.

Functional descriptions will also take a lead role in descriptions. Simply understanding the purpose or use of a part adds an understanding that is lacking from a description of only shape. However, care must be taken to so that the functional description complements, rather than supplants, the shape descriptions. 
Geometric terms will of course be prevalent in all descriptions. The utility of these terms is twofold. First, these terms can describe multiple features with a single word. Second, every shape can be described in geometric primitives and so when other means of description fail, multiple geometric terms can be used.

We observed no useful analogical descriptions and so we believe they will take a minor role at best in clear descriptions. When used, analogies need to carefully establish what features in the target are related to features in the host.

\section{Conclusion}

The data in this study support three conclusions relevant to teaching engineering:

1. The students were not able to verbally describe physical parts very well, and hence verbally communicating ideas within a team would be correspondingly weak. Though the descriptions included episodes of clarity, there were longer and more frequent periods where the descriptions were missing key elements to make interpretation straightforward.

2. The periods of clarity in the descriptions suggest that a curriculum could be constructed to explicitly teach verbal description. These data suggest that such a curriculum should include how to structure a description overall, how to describe details within the structure, and several examples of technical vocabulary to employ.

3. The students encountered the greatest difficulty describing the parts when they did not understand how the parts functioned. At times the students would substitute describing how they saw the parts working for what the parts were. This phenomenon suggests a cognitive link between understanding function and describing form that may be worthy of further study.

1 ABET Outcomes, Criterion 3. Retrieved 12/18/15 from http://www.abet.org/accreditation/accreditationcriteria/criteria-for-accrediting-engineering-programs-2016-2017/\#outcomes

2 Giesecke, F., Mitchell, A., Spencer, H., Hill, I., Dygdon, J., Novak, J., \& Lockhart, S., (2009). Technical Drawing, $13^{\text {th }}$ ed., Pearson Prentice Hall, Upper Saddle River, New Jersey.

3 Ullman, D., (1994). The Mechanical Design Process, $3^{\text {rd }}$ ed. McGraw-Hill, Boston, MA

${ }^{4}$ Zemke, S. \& Zemke, D., (2013). Cognitive hindrances to learning mechanical design. International Journal of Engineering Education 29(2): 450-458.

${ }^{5}$ Eckert, C. \& Stacey, M. (2001). Dimension of communication in design, International Conference on Engineering Design, Glasgow, Scotland.

${ }^{6}$ Bucciarelli, L. (2002). Between thought and object in engineering design, Design Studies, 23(3), 219-231.

7 Wiegers, T., Langeveld, L. \& Vergeest, J. (2011). Shape language: How people describe shapes and shape operations, Design Studies, 32(4), 333-347.

${ }^{8}$ Schön, D. (1987) Educating the Reflective Practitioner. Jossey-Bass, San Francisco.

9 Bransford, J. D., Brown, A. L., \& Cocking, R. R. (2000). How People Learn. Washington D.C., National Academy Press.

${ }^{10}$ Schön, D. (1983). The Reflective Practitioner. Basic Books, London.

11 Babbie, E. (2007). The Practice of Social Science Research (1 ${ }^{\text {th }}$ ed). Belmont, CA: Thomson-Wadsworth. 
${ }^{12}$ Leydens, J., Moskal, B., \& Pavelich, M. (2004). Qualitative methods used in the assessment of engineering education. Journal of Engineering Education, 93(1), 65-72.

13 Glesne, C. (2006) Becoming Qualitative Researchers ( $3^{\text {rd }}$ ed). San Francisco, CA: Pearson Education.

${ }_{14}$ Merriam (S). and associates (2002). Qualitative Research in Practice. San Francisco: Jossey-Bass.

${ }^{15}$ Stake, R., (1995). The Art of Case Study Research. Thousand Oaks, CA: Thousand Oaks, CA: Sage.

${ }^{16}$ Krippendorff, K. (2004). Content Analysis: An Introduction to its Methodology, Thousand Oaks, CA: Sage.

17 Elo, S. \& Kyngäs,H. (2008). The qualitative content analysis process, Journal of Advanced Nursing, 62(1), 7115.

${ }^{18}$ Hsieh. H. \& Shannon, S. (2005). Three approaches to qualitative content analysis, Qualitative Health Research, 15(9), 1277-1288. 